\title{
Regarding the article "The p160 ER co-regulators predict outcome in ER negative breast cancer" by Spears, Oesterreich, Migliaccio et al. published in Breast Cancer Res Treat, March 2011
}

\author{
Sara Alkner
}

Received: 19 April 2011/Accepted: 5 May 2011/Published online: 20 May 2011

(C) Springer Science+Business Media, LLC. 2011

To the Editor,

First we would like to congratulate the authors to an interesting study. However, when reading the article we wonder if the authors have missed our study "AIB1 is a predictive factor for tamoxifen response in premenopausal women", by Alkner S, Bendahl P-O, Grabau D, Lövgren $\mathrm{K}$, Stål O, Rydén L, Fernö M, published in Annals of Oncology 2009. We investigated AIB1 as a prognostic and treatment predictive factor using a controlled trial with premenopausal women randomized to receive tamoxifen for 2 years versus control. Randomization was done regardless of ER status. In line with the study by Spears et al. we found AIB1 to be a negative prognostic factor in patients not receiving tamoxifen. Subgroup analysis was done in relation to ER status, and the prognostic effect of AIB1 was then only significant in patients with ER-positive tumours (RFS: $\mathrm{HR}=1.8,95 \%$ CI 1.1-3.0, $P=0.02)(\mathrm{OS}$ : $\mathrm{HR}=1.9, \quad 95 \% \quad \mathrm{CI} 1.1-3.3, P=0.01)$, but not in ER-negative (RFS: HR $=1.5,95 \%$ CI $0.6-4.1, P=0.4$ )
$(\mathrm{OS}: \mathrm{HR}=1.2,95 \%$ CI $0.5-3.1, P=0.6)$. However, these differences in AIB1 effects between ER-positive and ER-negative patients were not significant when using a Cox model with a term for interaction. We also found ER-positive patients with a high AIB1 to respond very well to tamoxifen, increasing both RFS and OS to the same levels as in patients with low AIB1. This could explain why AIB1 was not associated to outcome in ER-positive tamoxifen treated patients in the study by Spears et al.

Since our study and the results presented in the published article in many ways are correlated to what was found by Spears et al., we would like to make the authors aware of this study. Also, the statement in their article that previous studies have not performed analyses separately for ER-positive and ER-negative disease is incorrect.

Best regards,

Sara Alkner, on behalf of all authors to our article "AIB1 is a predictive factor for tamoxifen response in premenopausal women". 